\title{
Prospective Evaluation of XRCC-1 as a Biopredictor for Survival Outcomes in Patients of Head and Neck Squamous Cell Carcinoma (HNSCC): A Meta-analysis
}

\section{Fan Yang}

Department of Otorhinolaryngology, Union Hospital, Tongji Medical College, Huazhong University of Science and Technology, Wuhan 430022 Liu-qing Zhou

Department of Otorhinolaryngology, Union Hospital, Tongji Medical College, Huazhong University of Science and Technology, Wuhan 430022 Yan-jun Wang ( $\nabla$ yjwang@hust.edu.cn )

Department of Otorhinolaryngology, Union Hospital, Tongji Medical College, Huazhong University of Science and Technology, Wuhan 430022

\section{Research Article}

Keywords: XRCC1, HNSCC, Prognosis, Meta-analysis

Posted Date: July 15th, 2021

DOI: https://doi.org/10.21203/rs.3.rs-698406/v1

License: @ (1) This work is licensed under a Creative Commons Attribution 4.0 International License. Read Full License 


\section{Abstract}

Background: Epidemiologic studies have demonstrated that X-ray repair cross-complementary group 1 (XRCC1) is one of the susceptibility factors in head and neck squamous cell carcinoma(HNSCC) patients. However, its clinical prognostic impact remains controversial. Thus, a meta-analysis was performed to clarify the survival value of XRCC1 in HNSCC patients.

Methods: Following the Preferred Reporting Items or Systematic Reviews Meta Analyses (PRISMA) 2020 guidelines, literature searches were systematically performed by PubMed, EMBASE and Web of Science with a manual retreive to evaluate the prognostic consequence of XRCC1 in HNSCC patients. Hazard ratios (HRs) and 95\% confidence intervals (Cls) were collected to estimate the correlation between XRCC1 and the survival outcomes of HNSCC patients.

Results: Ten studies including 2086 HNSCC patients who satisfied the inclusion and exclusion criteria were included in this meta-analysis. The meta-analysis showed that high XRCC1 expression and Arg399GIn and Arg194Trp were significantly correlated with poorer overall survival (OS), with HRs of $1.97(95 \% \mathrm{Cl}, 1.36-2.84, \mathrm{P}<0.001), 1.30$ (95\% Cl, 1.12-1.51, $\mathrm{P}<0.001)$, and $1.65(95 \% \mathrm{Cl}, 1.18-2.32 \mathrm{P}<0.05)$, respectively.

Conclusion: XRCC1 was associated with poorer survival outcomes in HNSCC patients. Hence, XRCC1 is a potential therapeutic target for HNSCC.

\section{Introduction}

Head and neck squamous cell carcinomas (HNSCC) is the fifth most common cancer in the world and accounts for approximately $3 \%$ of new cancer cases annually. It is a malignant disease of the upper aerodigestive tract, including oral, pharyngeal and laryngeal regions. Current treatment strategies for patients with HNSCC involve surgery, chemotherapy, immunotherapy, radiotherapy and so on. However, with such treatments, the survival results of HNSCC patients were still not satisfactory. The survival of HNSCC patients remain unsatisfactory. Specifically, the five-year survival rate of HNSCC patients is estimated to be near $50 \%$ due to high mortality and early disease recurrence. Therefore, there is an urgent need to identify new predictive biomarkers to improve the overall survival (OS) of HNSCC patients. Epidemiologic data showed that tobacco and alcohol consumption are the main etiological factors in the carcinogenesis of HNSCC. These risk factors, such as benzo(a)pyrene from tobacco, can induce an increase in reactive oxygen species levels that leads to oxidative DNA damage in the epithelial cells of the head and neck region, which stimulates HNSCC occurrence. However, not all individuals with these risk factors develop HNSCC, suggesting that individual susceptibility might also play a role in HNSCC carcinogenesis.

The DNA repair pathway can generally correct base lesions. In contrast, defects in the DNA repair system can also result in genetic instability and carcinogenesis. Single-nucleotide polymorphisms (SNPs) are genetic variants that are related to cancer susceptibility[1]. Hence, common genetic polymorphisms in genes involved in DNA repair capacity may play key roles in individual susceptibility and prognosis in HNSCC.

The primary DNA repair pathway that corrects base lesions is the base excision repair. X-ray repair cross-complementary group 1 (XRCC1) is a master DNA repair gene involved in base excision repair[2]. The capacity to repair damaged cells with XRCC1 is encoded by polymorphic genes that may modify the expression of encoded proteins and proteins. Consequently, the DNA repair ability is modified. Recently, XRCC1 gene SNPs within conserved sequences have been identified. Arg399GIn ( $r$ 25487) and Arg194Trp (rs1799782) are the main variant alleles [3, 4].

A number of studies have investigated the association between XRCC1 polymorphisms and HNSCC risk. However, the association between XRCC1 SNPs or expression and the survival outcomes in HNSCC patients is still largely controversial or unknown. Thus, a meta-analysis including a total of 10 studies with 2086 patients was performed by systematically searching for relevant publications from the PubMed, EMBASE, and Web of Science databases to provide a systematic evaluation of the diagnostic and prognostic role of XRCC1 polymorphisms and expression in HNSCC patients.

\section{Methods}

\section{Search strategy}

Comprehensively searches in PubMed, Web of Science and EMBASE were performed using the following search terms: (XRCC1 or X-ray repair cross-complementing 1) and (prognosis OR outcome OR mortality OR survival OR progression OR recurrence) and (head and neck or laryngeal or tonsil or oropharyngeal or oral or oropharynx or nasopharyngeal) and (squamous cell cancer or carcinoma). Related papers published from September 2006 to March 2021 were selected.

\section{Selection criteria}


The inclusion criteria of the meta-analysis were as follows: 1) all patients diagnosed with HNSCC; 2) the study revealed the association between XRCC1 and survival of HNSCC; 3) selected the English language of publications only; 4) including hazard ratios (HR) with prognostic endpoints and the $95 \%$ confidence interval(Cl) directly, or other data such as Kaplan- Meier survival curves that can be estimate for OS. The criteria of exclusion included: 1) studies without exsiting HR and 95\% Cl data for meta-analysis, or other outcomes for OS such as KaplanMeier survival curves. 2) Other text formats: abstracts, reviews, letters and case reports; and 3) The data of the article has nothing to do with HNSCC or XRCC1. In case of the same cohort reported by several publications, Latest literature gained the priority .

\section{Data extraction}

Following the Preferred Reporting Items or Systematic Reviews Meta Analyses (PRISMA) 2021 guidelines [5], electronic searches for relevant studies were performed in PubMed, EMBASE and Web of Science databases till March 1, 2021. Data were carefully extracted and tabulated by two independent researchers (LQ Zhou and Fan Yang) to minimize variation. In order to achieve a consensus, disagreements were solved by adjustments of the third researcher(YJ Wang). The following information was extracted from the studies: author, year, country, ethnicity, cancer type, sample size, age, follow-up, method, and HR value. The Newcastle-Ottawa Scale (NOS) was included to assess the quality of the included publications, and a star system (maximum is nine stars) was adopted to evaluate a study in three domains: comparability of study groups, selection of participants and ascertainment of outcomes of interest. Scores of NOS $\geq 6$ indicated high-quality studies[6]. Reporting recommendations for tumor marker prognostic studies (REMARK) were also applied to evaluate study quality in cancer-related metaanalyses[7].

\section{Statistical analysis}

The value of survival outcome between XRCC1 polymorphisms or expression and HNSCC was measured by $\mathrm{HR}$ and $95 \% \mathrm{Cl}$, which were obtained directly or estimated from the primary publications or estimated by $p$ values and other published data following Parmer's methods[8]. Heterogeneity was assessed by the $\chi^{2}$-based $Q$ test and the $\mathrm{I}^{2}$ test. The fixed-effects model was employed for analysis without obvious statistical heterogeneity between studies $\left(\mathrm{P}>0.10, \mathrm{I}^{2}<50 \%\right)$. Otherwise, the random-effects model was applied. Moreover, we performed subgroup analysis to explore the potential source of heterogeneity. Sensitivity analysis was also enrolled to investigate the influence of each individual study on the overall pooled results. Publication bias was assessed by Begg's and Egger's. All of the statistical tests used in this metaanalysis were performed with Stata version 12.0 (StataCorp, College Station, TX, USA).

\section{Results}

\section{Study selection and characteristics}

As shown in Fig. 1, 231 published articles were identified by searching the electronic databases. Out of 231 studies, duplicates ( $\mathrm{n}=131$ ), reviews, abstracts and letters $(n=16)$ and the studies not related to the topics $(n=44)$ were excluded. The retrieved publications $(n=40)$ were further evaluated by reading their full texts, and 30 studies were discarded due to the lack of specific data regarding HNSCC or XRCC1. Finally, 10 studies with 2086 HNSCC patients were selected.

The characteristics of the enrolled studies were summarized in Table 1. Six for HNSCC, one for laryngeal squamous cell carcinoma (LSCC), one for oral squamous cell carcinomas (OSCC), one for nasopharyngeal carcinoma (NPC) and one for oropharyngeal squamous cell carcinoma (OPSCC) were included. Sample sizes of the publications ranged from 75 to 531 . Two publications enrolled $>500$ patients. XRCC1 SNPs were explored by polymerase chain reaction restriction fragment length polymorphism (PCR-RFLP) and immunohistochemical (IHC) staining of XRCC1 protein technology were applied for XRCC1 protein expression. In the original literature, half of the studies reported the $\mathrm{HR}$ and $95 \% \mathrm{Cl}$ directly, and the remaining studies only provided Kaplan-Meier survival curves. All of the publications' NOS scores were above 6 and the REMARK scores were between 13 and 16.

Table 1

Characteristics of the studies examined in the meta-analysis

\section{Association between XRCC1 and survival in HNSCC patients}

Ten articles with 2086 patients in the meta-analysis evluated the survival value of XRCC1 for patients with HNSCC. The pooled HR for high XRCC1 expression was $1.97(95 \% \mathrm{Cl}, 1.36-2.84, \mathrm{P}<0.001)$, for the XRCC1 Arg399GIn was $1.30(95 \% \mathrm{Cl}, 1.12-1.51, \mathrm{P}<0.001)$ and for the XRCC1 Arg194Trp was $1.65(95 \% \mathrm{Cl}, 1.18-2.32, \mathrm{P}<0.005)$. The heterogeneity between high XRCC1 expression and OS $\left(\mathrm{I}^{2}=16.1 \%, \mathrm{P}_{\text {heterogeneity }}=\right.$ 0.304) was slight, and moderate heterogeneity was noted between XRCC1Arg194Trp and OS. $\left(I^{2}=53.0 \%\right.$, $\left.P_{\text {heterogeneity }}=0.119\right)$, while the heterogeneity between XRCC1 Arg399GIn and OS was low $\left(I^{2}=46.2 \%\right.$, $\left.P_{\text {heterogeneity }}=0.115\right)$ (Fig. 2). 


\begin{tabular}{|c|c|c|c|c|c|c|c|c|c|c|}
\hline Author & Year & Country & Ethnicity & $\begin{array}{l}\text { Cancer } \\
\text { type }\end{array}$ & $\begin{array}{l}\text { Sample } \\
\text { size }\end{array}$ & Age & $\begin{array}{l}\text { Follow-up } \\
\text { amonth }\end{array}$ & Method & HR & $\begin{array}{l}\text { NOS/REMARK } \\
\text { score }\end{array}$ \\
\hline Azad [9] & 2012 & Canada & $\begin{array}{l}\text { Non- } \\
\text { Asian }\end{array}$ & HNSCC & 531 & $63(33-86)$ & 100.52 & $\begin{array}{l}\text { PCR- } \\
\text { RFLP }\end{array}$ & Reported & $7 / 14$ \\
\hline Costa[2] & 2016 & Brazil & $\begin{array}{l}\text { Non- } \\
\text { Asian }\end{array}$ & OPSCC & 200 & $57(33-85)$ & $\begin{array}{l}24.581 .5- \\
116.7 区\end{array}$ & RT-PCR & Reported & $7 / 16$ \\
\hline Hirakawa [4] & 2020 & Japan & Asian & HNSCC & 225 & 67ه41-91】 & 48ه3-146】 & $\begin{array}{l}\text { PCR- } \\
\text { RFLP }\end{array}$ & Estimated & $6 / 14$ \\
\hline Jin [10] & 2014 & China & Asian & NPC & 75 & $45(22-72)$ & $25(5-46)$ & $\mathrm{IHC}$ & Reported & $7 / 14$ \\
\hline Fandino [1] & 2006 & Spain & $\begin{array}{l}\text { Non- } \\
\text { Asian }\end{array}$ & HNSCC & 103 & $\begin{array}{l}60.09(39.09- \\
94.00)\end{array}$ & $22.5(9-51)$ & $\begin{array}{l}\text { PCR- } \\
\text { RFLP }\end{array}$ & Estimated & $8 / 15$ \\
\hline Ang [11] & 2011 & America & $\begin{array}{l}\text { Non- } \\
\text { Asian }\end{array}$ & HNSCC & 77 & $56(39-61)$ & 66ه39-87】 & $\mathrm{IHC}$ & Reported & $7 / 14$ \\
\hline Bold [12] & 2021 & German & $\begin{array}{l}\text { Non- } \\
\text { Asian }\end{array}$ & HNSCC & 519 & NR & 60 & $\mathrm{IHC}$ & Estimated & $7 / 14$ \\
\hline Wang1 [13] & 2021 & China & Asian & oscc & 98 & $51(31-76)$ & $\begin{array}{l}40(2.4- \\
137.4)\end{array}$ & $\mathrm{IHC}$ & Reported & $8 / 16$ \\
\hline Wang2 [13] & 2021 & China & Asian & oscc & 98 & $51(31-76)$ & $\begin{array}{l}40(2.4- \\
137.4)\end{array}$ & $\mathrm{IHC}$ & Reported & $7 / 15$ \\
\hline Csejtei [3] & 2009 & Hungary & $\begin{array}{l}\text { Non- } \\
\text { Asian }\end{array}$ & HNSCC & 108 & 56.7 & 60 & $\begin{array}{l}\text { PCR- } \\
\text { RFLP }\end{array}$ & Estimated & $6 / 13$ \\
\hline Raturi [14] & 2020 & Japan & Asian & LSCC & 150 & $56(32-64)$ & 33 & $\begin{array}{l}\text { PCR- } \\
\text { RFLP }\end{array}$ & Reported & $6 / 14$ \\
\hline
\end{tabular}

\section{Sensitivity analysis}

During the process of comprehensive comparisons, we conducted sensitivity analyses to evaluate the effect of each single data point against the aggregate. The recalculated outcomes (Fig. 3)were not substantially influenced, suggesting that the combined effect size of the metaanalysis results was stable and reliable.

\section{Publication bias}

Begg's funnel plots and Egger's test were applied to validate potential bias from searched publications. The result indicates bias existed $(p=$ 0.014). Therefore, "trim and fill" analysis was further utilized, and the pooled HR of 1.253 (95\% Cl: 1.115-1.408) remained statistically significant (Figure. 4),overall, the results are relatively reliable in spite of the significant publication bias.

\section{Discussion}

Head and neck cancers are a heterogeneous group of secondary cancers[9], with a high rate of early recurrence and mortality. However, radiotherapy and chemotherapy have been widely used in the clinic and have recently been shown to be effective. The survival rates of advanced HNSCC are approximately $50 \%$ and have not changed significantly in recent years. This may be due to the individual's treatment response controlled by intrinsic genetics. In a previous study, oncogene genes in HNSCC were identified through extensive DNA sequencing and genetic analysis[10]. Therefore, it is reasonable to quest potential biomarkers of treatment by analyzing genomic features.

DNA repair genes have been considered driver genes of HNSCC due to their frequent mutation. Defects in DNA repair promote genomic instability and carcinogenesis [11]. DNA repair systems play an indispensable role in protecting cells against carcinogenic agents from internal and external stimuli[12]. Statistical analyses indicated that the DNA repair status of a tumor is associated with poor prognosis in cancer [13, 14]. XRCC1 is a major DNA repair gene involved in base excision repair (BER) for small base lesions resulting from oxidation and alkylation damage[15, 16]. More than 300 validated SNPs in the XRCC1 gene were reported in the dbSNP database (http://www.ncbi.nlm.nih.gov/SNP). Among them, the XRCC1 genes Arg194Trp and Arg399GIn, which occur within conserved sequences, are the most frequently mutated genes and the most extensively studied.

Studies have investigated the association between XRCC1 Arg399GIn/XRCC1 Arg194Trp polymorphisms and HNSCC risk [17-23]. However, these results were contradictory. Wang [24] conducted a meta-analysis on the association of XRCC1 Arg399GIn polymorphisms with HNSCC risk. Nevertheless, they did not observe any precise estimation of this relationship based on 18 published studies. Sturgis [25] reported a reduced risk between XRCC1 Arg194Trp and HNSCC while Andrew[26] found a weak elevation between HNSCC risk and the XRCC1 Arg194Trp polymorphism. XRCC1 protein is involved in BER $[27,28]$, and its protein expression alters the sensitivity of cells to radiation and 
chemotherapeutic agents [29]. It was reported that high protein expression levels of XRCC1 may be a risk factor for HNSCC [30, 31]. In addition, XRCC1 protein expression is common in HNSCC, and high XRCC1 protein expression may confer poorer survival, regardless of the primary tumor site or stage [31,32]. In summary, these results either contrast with each other or are not accurate conclusions.

In addition, this systematic review or meta-analysis prospectively evaluated XRCC1 as a biopredictor of survival outcomes in patients with HNSCC. There is no doubt that our study is the first meta-analysis including 10 published studies with 2086 patients to comprehensively evaluate the value of XRCC1 (SNPs and protein expression) and offer useful information for clinical decision-making in HNSCC.

After analyzing and summarizing all of the selected data, the results indicate that high expression and SNPs (Arg194Trp and Arg399GIn) of XRCC1 significantly predicted poorer OS in HNSCC patients with HRs of $1.97,1.65$, and 1.30, respectively. These findings confirmed that XRCC1 could be widely applied as a diagnostic marker and therapeutic target in HNSCC patients. As a genetic association literature, the HardyWeinberg (HWE) principle is also considered to avoid methodological weaknesses, such as biased selection of subjects or genotyping errors. The enrolled studies were all in agreement with HWE.

This meta-analysis should be interpreted within the context of its limitations. First, studies containing only English languages and only published articles were included, which may contribute to additional bias. Therefore, publication bias is very likely to occur. In light of Begg's funnel plots and Egger's test, no significant publication bias was present in this meta-analysis. Second, the number of articles was limited, and the sample size was relatively small in the present meta-analysis. False-positive or false-negative findings may have occurred in small sample sizes. Therefore, our results of the survival value in analyses might have occurred by chance, and more large-scale and comprehensive studies are needed to achieve a more persuasive conclusion. Third, the method of HR extrapolation from the Kaplan-Meier graph may also generate heterogeneity because the HRs of the five records enrolled were calculated by Parmer's methods and not directly reported. Last, studies with positive results are more likely to be published and thus more likely to be enrolled. Hence, the survival value of XRCC1 in HNSCC may to some extent have been overestimated in this meta-analysis.

In summary, our systematic review or meta-analysis was conducted to identify XRCC1 from all primary relevant articles and to evaluate the potential prognostic value of XRCC1 in HNSCC. The results demonstrated that both XRCC1 polymorphisms (Arg194Trp and Arg399GIn) and XRCC1 protein high expression exhibited poor survival in HNSCC patients. The prognostic value of XRCC1 in HNSCC patients is undoubtedly known. However, larger prospective studies should be conducted in the future to further verify the outcomes from this current meta-analysis

\section{Declarations}

\section{Acknowledgements}

This study was supported by grants from the National 12th-Five Year Research Program of China (no.2012BAl12B02)and Health Commission of Hubei Province scientific research project (no.wj2021M250).

\section{Ethics approval and consent to participate:}

Not applicable.

\section{Consent for publication:}

I agree that the text and any pictures or videos published in the article will be freely available on the internet and may be seen by the general public. The pictures, videos and text may also appear on other websites or in print, may be translated into other languages or used for commercial purposes.

\section{Availability of data and materials:}

All data generated or analyzed during this study are included in this published article [and its supplementary information files].

\section{Competing interests:}

The author(s) declare(s) that they have no competing interests. 


\section{Funding:}

Not applicable.

\section{Authors' contributions:}

YF and LZ collected and analyzed the data, wrote the paper. LZ, YF and JW analyzed the data and wrote the paper. LZ and JW conceived and designed this study, analyzed the data, wrote the paper. All authors read and approved the final manuscript.

Acknowledgements: Not applicable.

\section{Data availability statements}

All data generated or analyzed during this study are included in this published article [and its supplementary information files].

The data that support the findings of this study are available from the corresponding author upon reasonable request.

\section{References}

1. Quintela-Fandino, M., et al., DNA-repair gene polymorphisms predict favorable clinical outcome among patients with advanced squamous cell carcinoma of the head and neck treated with cisplatin-based induction chemotherapy. J Clin Oncol, 2006. 24(26): p. 4333-9.

2. Costa, E.F., et al., Association between polymorphisms in genes related to DNA base-excision repair with risk and prognosis of oropharyngeal squamous cell carcinoma. J Cancer Res Clin Oncol, 2016. 142(9): p. 1917-26.

3. Csejtei, A., et al., Association between XRCC1 polymorphisms and head and neck cancer in a Hungarian population. Anticancer Res, 2009. 29(10): p. 4169-73.

4. Hirakawa, H., et al., ERCC1 C8092A polymorphism predicts fair survival outcome in Japanese patients with pharyngo-laryngeal squamous cell carcinoma. Eur Arch Otorhinolaryngol, 2020. 277(2): p. 601-610.

5. Page, M.J., et al., The PRISMA 2020 statement: An updated guideline for reporting systematic reviews. Int J Surg, 2021. 88: p. 105906.

6. Stang, A., Critical evaluation of the Newcastle-Ottawa scale for the assessment of the quality of nonrandomized studies in meta-analyses. Eur J Epidemiol, 2010. 25(9): p. 603-5.

7. Sauerbrei, W., et al., Reporting Recommendations for Tumor Marker Prognostic Studies (REMARK): An Abridged Explanation and Elaboration. J Natl Cancer Inst, 2018. 110(8): p. 803-811.

8. Parmar, M.K., V. Torri, and L. Stewart, Extracting summary statistics to perform meta-analyses of the published literature for survival endpoints. Stat Med, 1998. 17(24): p. 2815-34.

9. Azad, A.K., et al., Genetic sequence variants and the development of secondary primary cancers in patients with head and neck cancers. Cancer, 2012. 118(6): p. 1554-65.

10. Comprehensive genomic characterization of head and neck squamous cell carcinomas. Nature, 2015. 517(7536): p. 576-82.

11. Tubbs, A. and A. Nussenzweig, Endogenous DNA Damage as a Source of Genomic Instability in Cancer. Cell, 2017. 168(4): p. 644-656.

12. Leemans, C.R., B.J. Braakhuis, and R.H. Brakenhoff, The molecular biology of head and neck cancer. Nat Rev Cancer, 2011. 11(1): p. 9-22.

13. Birkbak, N.J., et al., Paradoxical relationship between chromosomal instability and survival outcome in cancer. Cancer Res, 2011. 71(10): p. 3447-52.

14. Azad, A.K., et al., Validation of genetic sequence variants as prognostic factors in early-stage head and neck squamous cell cancer survival. Clin Cancer Res, 2012. 18(1): p. 196-206.

15. Lou, Y., et al., DNA repair gene XRCC1 polymorphisms and head and neck cancer risk: an updated meta-analysis including 16344 subjects. PLoS One, 2013. 8(9): p. e74059.

16. Almeida, K.H. and R.W. Sobol, A unified view of base excision repair: lesion-dependent protein complexes regulated by post-translational modification. DNA Repair (Amst), 2007. 6(6): p. 695-711.

17. Kostrzewska-Poczekaj, M., et al., Polymorphisms of DNA repair genes and risk of squamous cell carcinoma of the head and neck in young adults. Eur Arch Otorhinolaryngol, 2013. 270(1): p. 271-6.

18. Gugatschka, M., et al., DNA repair gene ERCC2 polymorphisms and risk of squamous cell carcinoma of the head and neck. Exp Mol Pathol, 2011. 91(1): p. 331-4. 
19. Li, C., et al., Genetic polymorphisms in DNA base-excision repair genes ADPRT, XRCC1, and APE1 and the risk of squamous cell carcinoma of the head and neck. Cancer, 2007. 110(4): p. 867-75.

20. Applebaum, K.M., et al., Smoking modifies the relationship between XRCC1 haplotypes and HPV16-negative head and neck squamous cell carcinoma. Int J Cancer, 2009. 124(11): p. 2690-6.

21. Majumder, M., et al., Polymorphisms at XPD and XRCC1 DNA repair loci and increased risk of oral leukoplakia and cancer among NAT2 slow acetylators. Int J Cancer, 2007. 120(10): p. 2148-56.

22. Jin, H., et al., ERCC1 Cys8092Ala and XRCC1 Arg399GIn polymorphisms predict progression-free survival after curative radiotherapy for nasopharyngeal carcinoma. PLoS One, 2014. 9(7): p. e101256.

23. Raturi, V., et al., Prospective evaluation of XRCC-1 Arg194Trp polymorphism as bio-predictor for clinical outcome in locally advanced laryngeal cancer undergoing cisplatin-based chemoradiation. Head Neck, 2020. 42(5): p. 1045-1056.

24. Wang, Y., et al., Association of X-ray repair cross complementing group 1 Arg399GIn polymorphisms with the risk of squamous cell carcinoma of the head and neck: evidence from an updated meta-analysis. PLoS One, 2013. 8(10): p. e77898.

25. Sturgis, E.M., et al., Polymorphisms of DNA repair gene XRCC1 in squamous cell carcinoma of the head and neck. Carcinogenesis, 1999. 20(11): p. 2125-9.

26. Olshan, A.F., et al., XRCC1 polymorphisms and head and neck cancer. Cancer Lett, 2002. 178(2): p. 181-6.

27. Thompson, L.H. and M.G. West, XRCC1 keeps DNA from getting stranded. Mutat Res, 2000. 459(1): p. 1-18.

28. Weaver, D.A., et al., ABCC5, ERCC2, XPA and XRCC1 transcript abundance levels correlate with cisplatin chemoresistance in non-small cell lung cancer cell lines. Mol Cancer, 2005. 4(1): p. 18.

29. Park, S.Y., W. Lam, and Y.C. Cheng, X-ray repair cross-complementing gene I protein plays an important role in camptothecin resistance. Cancer Res, 2002. 62(2): p. 459-65.

30. Wang, Y.Y., et al., Excision repair cross-complementing group 2 upregulation is a potential predictive biomarker for oral squamous cell carcinoma recurrence. Oncol Lett, 2021. 21(6): p. 450.

31. Ang, M.K., et al., High XRCC1 protein expression is associated with poorer survival in patients with head and neck squamous cell carcinoma. Clin Cancer Res, 2011. 17(20): p. 6542-52.

32. Bold, I.T., et al., DNA Damage Response during Replication Correlates with CIN70 Score and Determines Survival in HNSCC Patients. Cancers (Basel), 2021. 13(6).

\section{Figures}

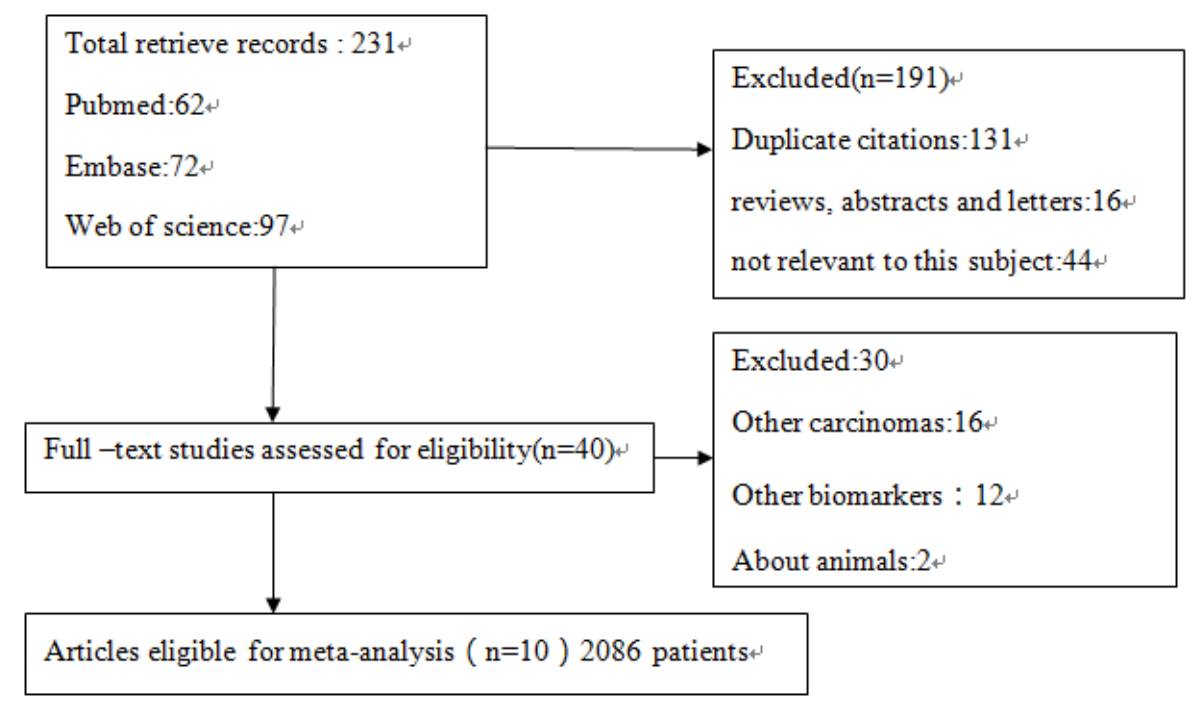

\section{Figure 1}

Flow diagram of the selection of relevant studies included in the meta-analysis 


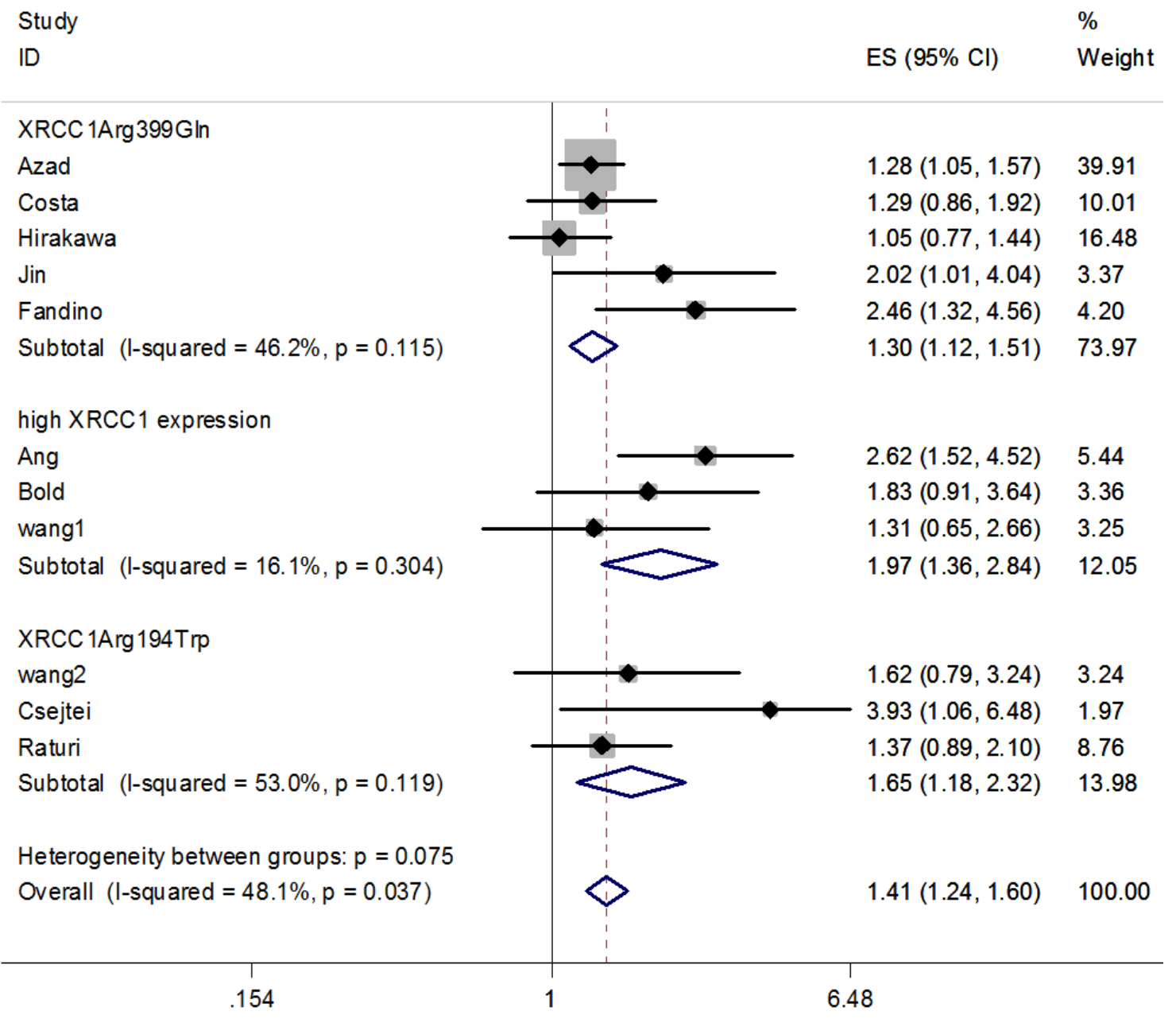

\section{Figure 2}

Forest plot indicating the association between XRCC1 polymorphisms (Arg194Trp and Arg399GIn) / high XRCC1 protein expression and OS in HNSCC

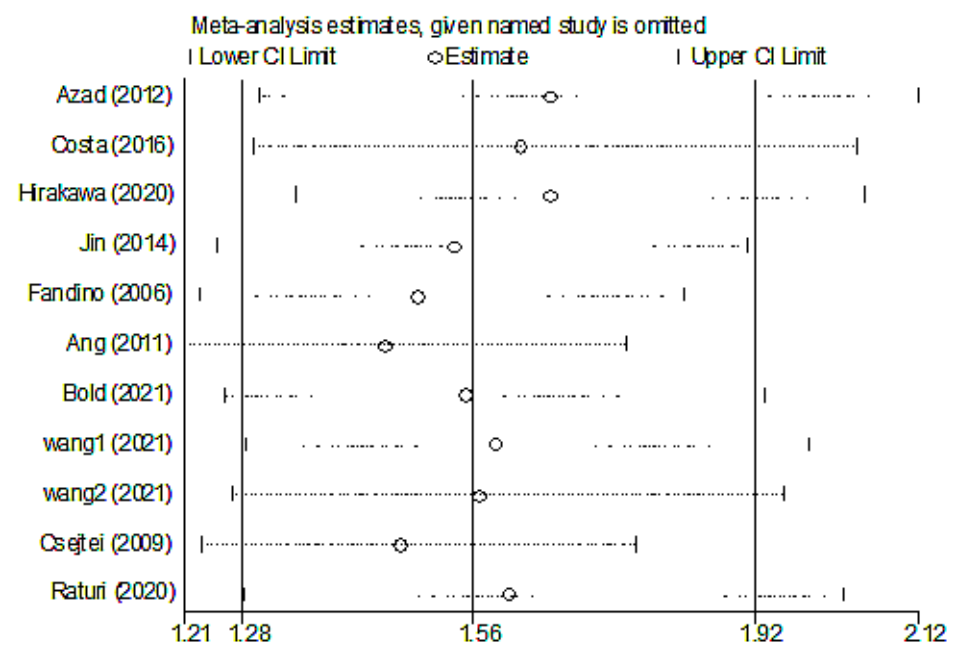

Figure 3 
The sensitivity analyses were conducted to evaluate the effects of each single study on the overall effect

a

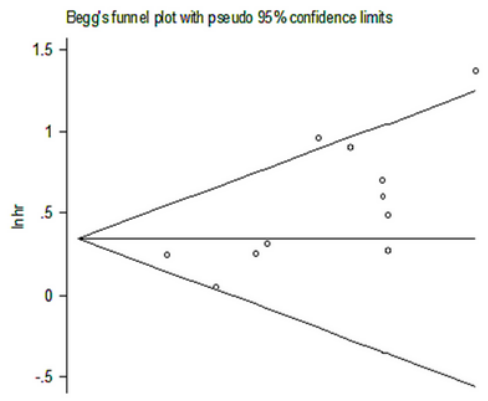

b

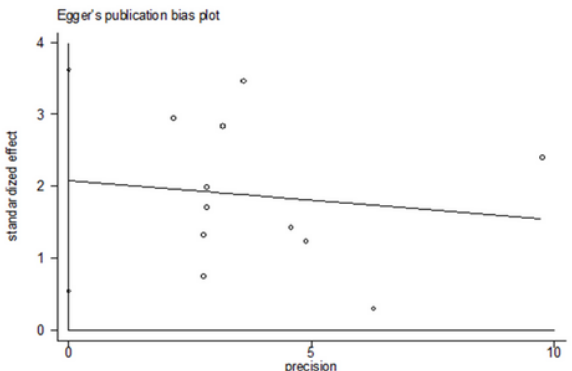

C

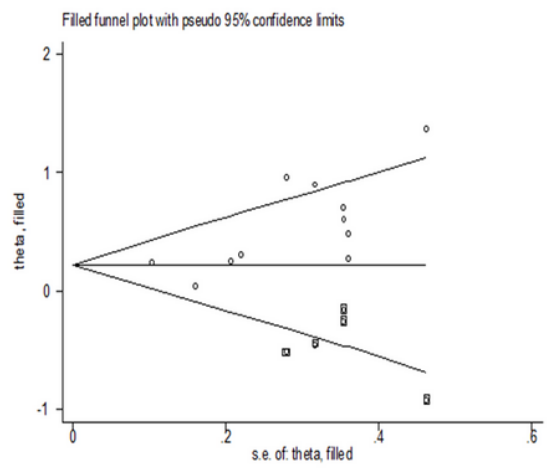

\section{Figure 4}

Publication bias and trim and fill analysis of the enrolled analysis. a The Begg's funnel plots; b The Egger's test. c Trim and fill analysis 